\title{
RAYSAR - 3D SAR SIMULATOR: NOW OPEN SOURCE
}

\author{
Stefan Auer ${ }^{1}$, Richard Bamler ${ }^{1,2}$, Peter Reinartz ${ }^{1}$ \\ ${ }^{1}$ Remote Sensing Technology Institute, German Aerospace Center (DLR), Oberpfaffenhofen, Germany \\ ${ }^{2}$ Chair of Remote Sensing Technology, Technische Universität München, Germany
}

\begin{abstract}
RaySAR, a 3D SAR simulator, has been made accessible to the SAR community in January 2016. It helps to understand and analyze reflection effects of radar signals at 3D object models. For instance, the nature of persistent scatterers or "ghost" scatterers at man-made structures or basic reflection effects at canonical shapes can be interpreted in more detail. The decision to provide RaySAR to the community was based on the persisting lack of a freely available SAR simulation package and the repetitive interpretation task related to the analysis of SAR imagery, especially in the context of high resolution data. Many signal reflection effects at objects remain to be discovered and explained. New interfaces between simulation techniques and SAR applications may be helpful. In this context, the paper provides a summary of the status-quo of RaySAR and addresses future directions that have not been followed so far.
\end{abstract}

Index Terms - SAR, SAR simulation, 3D Models, Interpretation, Ray Tracing, Open Source

\section{INTRODUCTION OF RAYSAR AND MOTIVATION}

RaySAR [1, 2] originates from interpretation problems in the context of persistent scatterer interferometry (PSI) and high resolution SAR imaging in X-Band (TerraSAR-X and TanDEM-X satellites) and was developed in a joint effort of DLR and the Technische Universität München in the course of a doctoral thesis. The basic aim was to understand the nature of scatterers which correspond to dominant and temporally persistent signatures in SAR images (e.g. isolated points or points arranged in patterns or along lines). In a nutshell, RaySAR, which is based on an extended version of the ray tracer POV-Ray, was developed to

- simulate image layers in the azimuthrange/ground range plane (SAR image, reflectivity map, separate layers for reflection levels 1-5, binary mask marking pixels containing signals related to specular reflections).

- simulate the positions of signal phase centers in azimuth/range/elevation or world coordinates, which supports the interpretation/evaluation of the results of 3D SAR algorithms (interferometry, tomography, radargrammetry).

- mark the extent of reflecting surfaces contributing to salient image pixels and analyze the physical correspondence to object bodies

The simulation package can be downloaded at https://github.com/StefanJAuer/RaySAR/releases

It includes the source code to set up RaySAR in Windows and Linux environments, documentation files and 3D models for test purposes. The programming language is MATLAB for image simulation and $\mathrm{C}++$ for the ray tracing part. Typically, the calculation time per scene object is in the range of minutes on standard PCs.

Alternative approaches in the literature are based, for instance, on ray tracing $[3,4]$ or rasterization on graphics card [5]. To the knowledge of the author, no SAR image simulation package is available for free and with open source code as yet.

RaySAR can be categorized as SAR image simulator, i.e. phase center positions and image layers are 

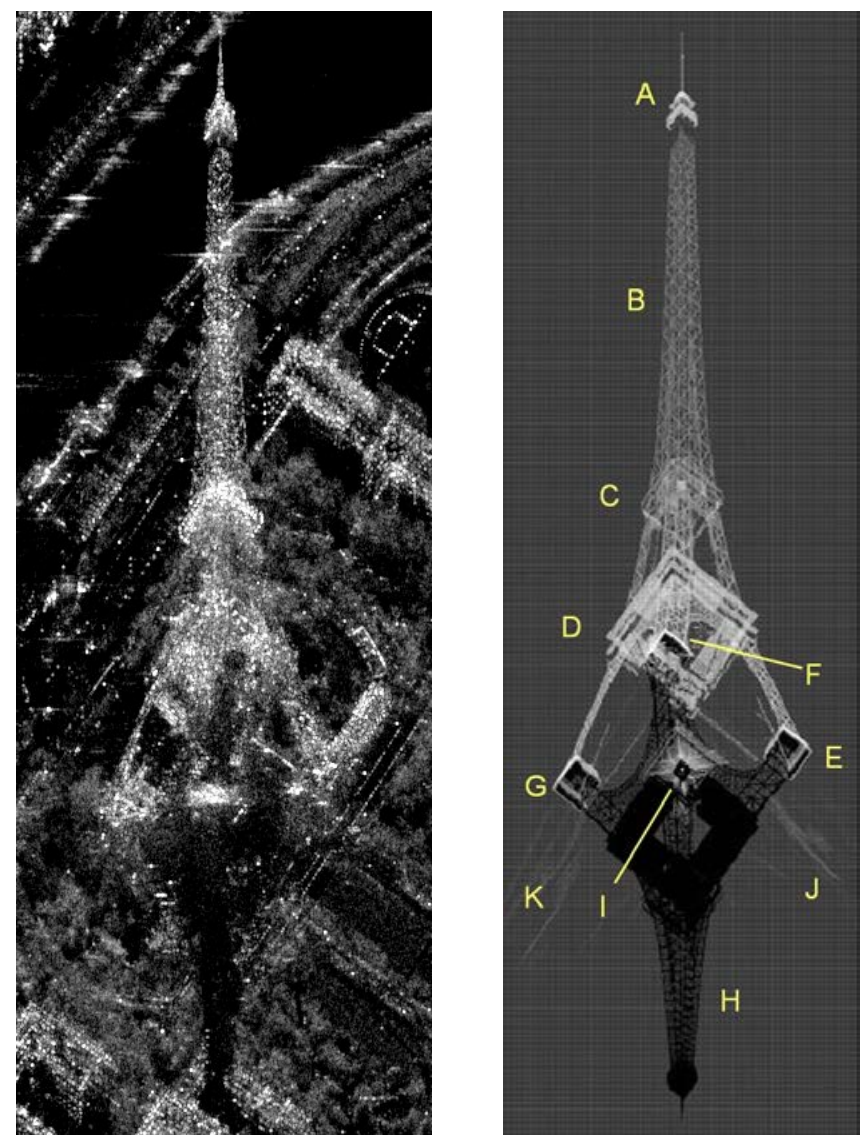

Fig. 1: Simulation of Eiffel Tower, Paris, France [2]. Left: TerraSAR-X image, Right: Image simulated by RaySAR. A: pinnacle, B: cross beam structures, C, D: plattforms; EFG: feet; H: shadow zone, I, J, K: (diffuse) signal double reflections.

provided without raw data simulation and processing. Focusing effects related to signal multiple reflections are calculated in the course of the ray tracing procedure. The effect of signal focusing is assigned artificially by diminishing the initial infinite spatial resolution (corner reflectors are focused in one point in 3D space). RaySAR has been applied for different purposes and various scene models, including 2.5D digital surface models (DSMs) without structural details and hand-crafted facade models with $\mathrm{cm}$ accuracy. Simulations have been performed in local or world coordinates (see case studies [2] and [6], respectively). As an example, Fig. 1 presents the simulation result for a detailed model of the Eiffel tower [2]. Fig. 2 shows the simulation result for a facade in Munich, Germany [6]. The 3D model has been reconstructed based on photogrammetric and geodetic methods (GPS, theodolite). Geometric details have been considered based on their relevance for
SAR imaging (corner reflectors with side lengths $>8$ $\mathrm{cm})$. The accuracy of the model is better than $2 \mathrm{~cm}$.

RaySAR has helped to gain knowledge in the context of 3D SAR algorithms, e.g. for explaining the nature of "ghost persistent scatterers" [7]. However, many signal reflection effects remain unexplained as the appearance of signatures in SAR images strongly varies with object type, scene arrangements and surface materials. Moreover, only a small section of possible simulation applications has been covered so far. For these reasons, the authors intend to increase the number of scientists using RaySAR. The following section may provide some guidelines.

\section{RAYSAR - STATUS QUO AND POSSIBLE EXTENSIONS / APPLICATIONS}

The strengths (geometry, full coverage of specular reflections, variability with respect to $2 \mathrm{D}$ and $3 \mathrm{D}$ SAR simulations and object models, speed in the context of signal multiple reflections) and limitations (simplified reflection models, missing polarimetric aspect, incompleteness of diffuse signal reflections) of RaySAR are well understood and discussed in [1]. This section aims at moving one step further. To start with, the status-quo of RaySAR can be summarized as follows:

- Software: RaySAR has been tested in the course of various case studies and continuously optimized in this context (simulation modules, modeling support, graphical user interfaces). The focus has mostly been on multi-body scenes, building shapes and facade structures. Besides, dedicated case studies have been conducted for bridges, railways, and trees with random foliage. The software is of experimental nature and offers various opportunities for enhancements.

- Formats: The following formats have been primarily used in the context of RaySAR simulations: 3D studio max (.3ds), Wavefront (.obj), and AutoCAD (.dxf). Canonical bodies have commonly been modeled using the POVRay editor. Different software alternatives are available for the translation of model formats into POV-Ray language (e.g. AccuTrans 3D), i.e. the flexibility of RaySAR w.r.t. model import is high. 
- Building models: The level of detail of object models is of major importance for the interpretation of object-related SAR image signatures. Moreover, the availability of absolute model coordinates (e.g. UTM) and information on the model accuracy has proven to be the backbone for simulations related to SAR applications [6] (e.g. for DSMs, detailed facade models). 3D models from the internet, in contrast, are often limited in their use, as relevant structural details are missing and the model origin is commonly not known.

- Reflection models: The importance of signal reflection models increases with the level of detail of the object model, i.e. the application of high-level reflection models is useless if object shapes are not fully represented by the 3D model (e.g. balcony or window structures for facades). RaySAR covers the angular dependence and proportion related to specular reflections, whereas the resulting amplitude values are artificial.

From the authors' viewpoint, upcoming applications / extensions of RaySAR may concentrate on the following directions:

- Reference data: 3D models of very high detail (see [6] as an example) allow for the simulation of reference positions in order to evaluate the relative / absolute accuracy of 3D SAR algorithms such as PSI, TomoSAR, and Radargrammetry. Even if first case studies have been conducted using RaySAR, many aspects have not been covered so far due to the variability of scene arrangements and object bodies.

- Phase center corrections: RaySAR simulates phase center positions as derived from 2D or 3D SAR algorithms. In that regard, it also considers the displacement of phase center positions related to signal multiple reflections [7]. Future applications may make use of this in order to elaborate strategies to map such phase centers ("ghost scatterers") to their true positions.

- Signal multiple reflections: RaySAR is restricted to reflection levels 1-5 in its current form. However, it is well known that higher

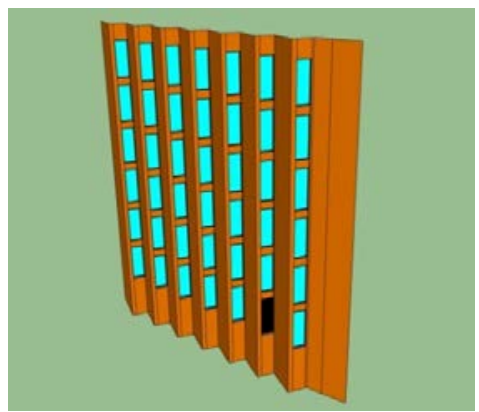

3D model

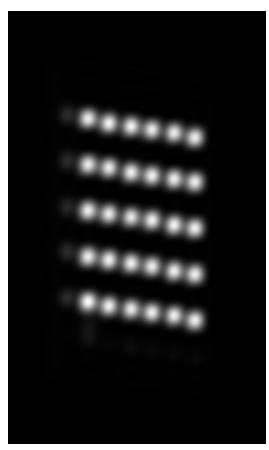

Simulated image in azimuth (increasing left-right) and range (increasing top-down)

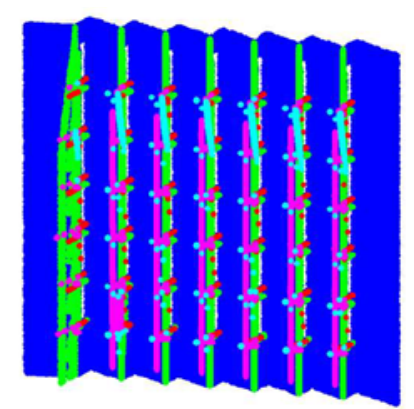

Distribution of signal components in azimuth/range plane (blue: single bounce, green: double bounce, red: triple bounce, magenta: fourfold bounce, cyan: fivefold bounce)

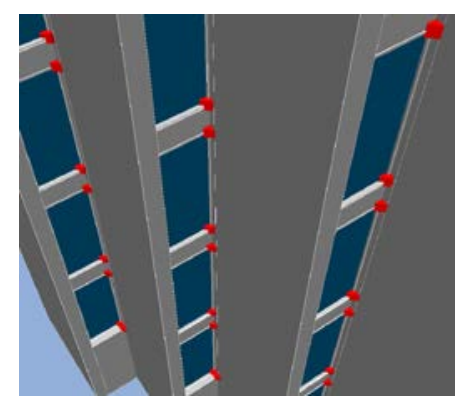

3D position of signal phase centers (marked in red)

Fig. 2: Simulation example for a facade in Munich City Center [6] 
reflection levels are apparent for specific scene arrangements, e.g. urban channels. Extending RaySAR to reflection level $\mathrm{X}>5$ is possible with little effort (by discarding the limit in the POV-Ray source code and adding the generation of $\mathrm{X}$ - 5 image layers in the MATLAB code). The restriction to reflection level $\mathrm{Y}<\mathrm{X}$ can then be imposed using the POV-Ray command "global_settings" (parameter “max_trace_level”).

- Reflection models: RaySAR would benefit from the use of advanced signal reflection functions in the context of high-level 3D models (global illumination, polarimetry).

- Processing chain: The image generation part of RaySAR can be integrated into processing chains (see [8] as an example), in order to automatically identify areas of interest in SAR images (e.g. layover, shadow, ground) in absolute coordinates. In this context, the interpretation step can be related to scene objects (using individual input models) and conducted independent of the SAR sensor perspective.

- Applications in other fields: RaySAR in its current form can be utilized for other tasks in the field of remote sensing / photogrammetry if the reflection level is limited to 1 (direct signal response). Examples may be:

o Complementary to the SAR simulation step, optical data are provided as standard output of POVRay. Several camera models are available and adaptable with parameters (e.g. orthographic, perspective, fisheye, spherical, cylindrical)

o Rendered optical images can be combined with depth information provided by RaySAR (for each pixel), which may be of interest in the context of terrestrial applications (e.g. range image systems).

o Artificial digital surface models (DSMs) can be generated for input models using an orthographic camera pointing in nadir direction, followed by the inversion of depth values obtained from the ray tracer.

\section{SUMMARY}

RaySAR, a 3D SAR simulation software based on ray tracing techniques, is now available to the SAR community (downloadable via GitHub). In order to ease its applicability, this paper has summarized the main properties and the current status of the software as well as promising directions for extensions / future enhancements.

\section{REFERENCES}

[1] S.J. Auer, "3D Synthetic Aperture Radar Simulation for Interpreting Complex Urban Reflection Scenarios”, TU München, 2011

[2] S. Auer, S. Hinz, R. Bamler, "Ray-tracing Simulation Techniques for Understanding Highresolution SAR Images”, IEEE Trans. Geosci. Remote Sens., 48 (3), 2010, 1445-1456

[3] H. Hammer, K. Schulz, "Coherent Simulation of SAR Images”, Proceedings of SPIE Image and Signal Processing for Remote Sensing XV, 2009, 7477, 74771K-1-8

[4] H. Anglberger, R. Speck, T. Kampf, H. Suess, "Fast ISAR Image Generation through Localization of Persistent Scattering Centers”, Proceedings of SPIE Defence, Security and Sensing, 2009, 7308

[5] T. Balz, U. Stilla, "Hybrid GPU-Based Single- and Double-Bounce SAR Simulation”, IEEE Trans. Geosci. Remote Sens., 2009, 47, 3519-3529

[6] S. Auer, S. Gernhardt, K. Eder, C. Gisinger, "Simulation of Reference Data for 3D SAR Algorithms”, IGARSS 2015, 2927-2930

[7] S. Auer, S. Gernhardt, R. Bamler, "Ghost Persistent Scatterers Related to Multiple Signal Reflections”, IEEE Geosci. Remote Sens. Lett., 2011, 8, 919-923

[8] J. Tao, S. Auer, G. Palubinskas, P. Reinartz, R. Bamler, "Automatic SAR Simulation Technique for Object Identification in Complex Urban Scenarios", IEEE J. Sel. Topics Appl. Earth Observ. in Remote Sens., 2014, 7, 994-1003 